initial MRI edema score and the level of serum CK $(p=0.028)$. However, no correlation was seen between the level of improvement in MRI ES and drop in CK or with any other clinical parameters after 3 months of treatment. The intensity of muscle edema at baseline was not predictive to improvement of any clinical parameters or CK, with a tendency to a better improvement of muscle disease activity in those who had higher MRI ES at baseline (corr. coeff. $0.445, \mathrm{p}=0.073$ ).

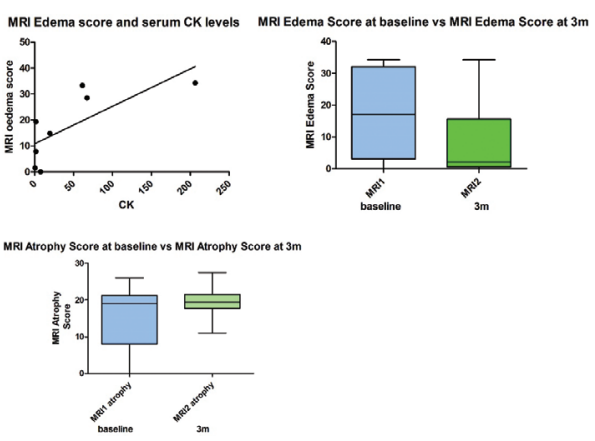

Conclusion: We proposed a new semi-quantitative scoring system for assessment of MR images in patients with PM and DM. This system was applied to record degree of muscle edema and fatty replacement in a subpopulation of patients treated in the Prometheus study. A significant reduction of MRI ES and no relevant progression of muscle atrophy and fatty replacement after 3 months of therapy was demonstrated. Initial MRI edema score correlated with some clinical and laboratory parameters (PGA, CK) but was not predictive to a degree of improvement during treatment. The results are limited by a small sample size, particularly in the subset with longitudinal MRI.Acknowledgement: Supported by the project (Ministry of Health, Czech Republic) for conceptual development of research organization 00023728 (Institute of Rheumatology).

Disclosure of Interests: Kateřina Kubinova: None declared, Jiří Vencovský Consultant for: Samsung, Speakers bureau: AbbVie, Novartis, Pfizer, Sanofi, Eli Lilly, Biogen, UCB, MSD, Werfen, Roche DOI: 10.1136/annrheumdis-2019-eular.7616

\section{FRI0643 SPINE AND SACROILIAC JOINTS LESIONS ON MRI IN PATIENTS WITH EARLY AXIAL SPA: CORRELATION WITH CLINICAL AND DISEASE ACTIVITY INDICES IN 24- MONTHS FOLLOW UP (ITALIAN ARM OF SPACE STUDY)}

Mariagrazia Lorenzin ${ }^{1}$, Augusta Ortolan ${ }^{1}$, Mara Felicetti ${ }^{1}$, Pamela Polito ${ }^{1}$, Stefania Vio ${ }^{2}$, Marta Favero ${ }^{1}$, Carmelo Lacognata ${ }^{2}$, Roberta Ramonda ${ }^{1}$.

${ }^{1}$ Rheumatology Unit, University Hospital of Padova, Department of MedicineDIMED, Padova, Italy, ${ }^{2}$ University Hospital of Padova, Radiology Unit, Padova, Italy

Background: Recently several studies have focused on the use of magnetic resonance imaging (MRI) that might facilitate early diagnosis and monitor the disease activity of axial spondyloarthritis (axSpA).

Objectives: Evaluate the prevalence of spine and pelvis MRI lesions in patients (pts) with low back pain (LBP) and suspected axSpA;investigate how MRI features evolve over time and relate to radiographic damage; identify the predictive factors for a more severe disease pattern with a higher probability of radiological progression.

Methods: Seventy-five pts with LBP $(>3$ months, $\leq 2$ years, onset $\leq 45$ years) underwent a physical examination, questionnaires, laboratory tests, X-rays and MRI of the spine and sacroiliac joints (SIJ) at baseline (T0) and during a follow-up (FU) period of 24 months. Two expert rheumatologists defined axSpA diagnosis and assessed fulfilment of Assessment of SpondyloArthritis International Society ASAS criteria.Disease activity and physical functioning were assessed using: Bath Ankylosing Spondylitis Metrology Index (BASMI):Maastricht Ankylosing enthesitis Spondilities Score (MASES);Bath Ankylosing Spondylitis Disease Activity Index (BASDAl):Bath Ankylosing Spondylitis Functional Index (BASFI):Ankylosing Spondylitis disease activity score (ASDAS);Visual Analogue Scale (VAS pain);VAS night pain;VAS disease activity;Bath Ankylosing Spondylitis Patient Global Score (BASG1);BASG2;Health Assessment Questionnaire (HAQ);ESR;serum ultrasensitive CRP (hs-CRP).Spine and SIJ MRI and X- rays were scored independently by 2 readers following the SPARCC, mSASSS and score SIJ-mNY.Pts were classified in accordance ASAS criteria as:21 pts classified according to axSpA imaging arm;29 pts classified according to axSpA clinicaltimaging arm;25 pts not fulfilling ASAS criteria.The Kruskal Wallis test was used to compare all indices during FU.A regression analysis was performed to identify the predictors of inflammation and radiological progression of the disease evaluated using mSASSS, score SIJ-mNY, SPARCC-SIJ and SPARCC-spine.The following independent variables were considered in this analysis:female sex,age of LBP onset,duration of LBP,presence of HLA-B27, elevated ESR/CRP, BASDAI $>4$, use of NSAIDs.

Results: The median LBP onset was $28.51 \pm 8.05$ years, $45.3 \%$ were male, HLA-B27 was positive in $38.7 \%$ pts; $56 \%$ pts showed bone marrow edema (BME) in spine-MRI $(19 \%, 39 \%, 33 \%$ in cervical/thoracic/lumbar region) and $61.3 \%$ pts in SIJ-MRI (respectively $58 \%$ on the right SIJ and $50 \%$ on the left SIJ).Signs of enthesitis were found in $62.7 \%$ pts (respectively $8 \%, 58 \%, 11 \%$ in the cervical/thoracic/lumbar spine).Eigtheen $(24 \%)$ pts presented a negative BME SIJ-MRI with a positive BME spine-MRI. Predictive factors of increased radiological activity were, respectively, for a higher SPARCC-spine score, a higher use of NSAIDs and for a higher SPARCC-SIJ score, the presence of HLA-B27 and increased ESR/CRP. The prevalence of BME lesions on MRI and SPARCC-SIJ decreased during the FU period in the 2 cohorts that met the ASAS criteria for axSpA (Table 1, Figure 1a-b).
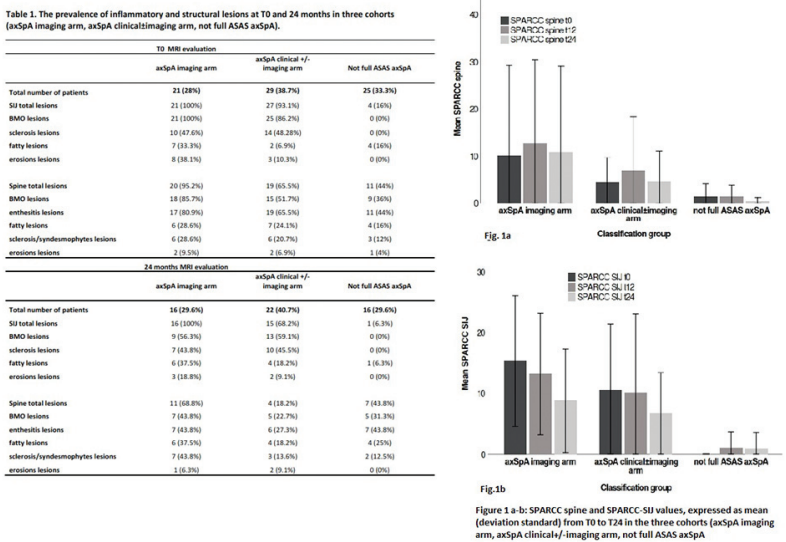

Conclusion: At TO a significant prevalence of BME lesions was observed both for SIJ and the spine, with predominant involvement of thoracic district.Since positive spine-MRI images were observed in absence of sacroiliitis, we can hyphothesize that this finding could have a diagnostic significance in suspected axSpA.Further studies are warranted to assess in a more exhaustive manner the role of imaging in the monitoring of activity disease and radiological progression.Disclosure of Interests: None declared

DOI: 10.1136/annrheumdis-2019-eular.2654

\section{FRI0644 ANALYSIS OF CARDIOVASCULAR RISK AND CAROTID INTIMA-MEDIA THICKNESS IN PATIENTS WITH PSORIASIS}

José Alexandre Mendonça ${ }^{1}$, Elaine Cristina Faria Abrahão Machado ${ }^{2}$, Luciana B. Nucci ${ }^{2}$, Ana Carolina Belini Bázan Arruda ${ }^{2} .{ }^{1}$ Pontifical Catholic University of Campinas, Rheumatology, Campinas, Brazil; ${ }^{2}$ Pontifical Catholic University of Campinas, Campinas, Brazil

Background: Psoriasis (Ps) is associated with atherosclerosis and an increased risk of cardiovascular disease (CVR). Currently, a new automated ultrasound software, based on radio frequency, called QIMT (quantitative intima media thickness) technology, proved to be a useful method for assessing subclinical atherosclerosis with the measurement of the intima-media thickness (IMT) in carotid arteries ${ }^{1,2}$

Objectives: To analyze the ultrasound results of the QIMT and Framingham score for psoriasis patients submitted to two types of treatments: methotrexate (MTX) and tumor necrosis factor inhibitor (TNF- $\alpha$ ).

Methods: Fifty patients with psoriasis in plaques, were divided into two groups, using MTX and using TNF- $\alpha$ (infliximab and adalimumab). We 